\title{
Design and Control of MIRA: a Lightweight Climbing Robot for Ship Inspection
}

\author{
Mohammed Ahmed, Markus Eich, Felix Bernhard \\ German Research Center for Artificial Intelligence (DFKI) Robotics Innovation Center, Bremen, \\ Germany \\ E-mail address: felix.bernhardg@dfki.de
}

Keywords: maritime robotics, wheeled robots, magnetic crawlers

\begin{abstract}
The inspection of marine vessels is currently per-formed manually. Inspectors use tools (e.g. cameras and devices for non-destructive testing) to detect damaged areas, cracks, and corrosion in large cargo holds, tanks, and other parts of a ship. Due to the size and complex geometry of most ships, ship inspection is time-consuming and expensive. The EU-funded project INCASS develops concepts for a marine inspection robotic assistant system to improve and automate ship inspections.

In this paper, we introduce our magnetic wall-climbing robot: Marine Inspection Robotic Assistant (MIRA). This semi-autonomous lightweight system is able to climb a vessels steel frame to deliver on-line visual inspection data. In addition, we describe the design of the robot and its building subsystems as well as its hardware and software components.
\end{abstract}

\section{INTRODUCTION}

Marine vessels, such as bulk carriers, dry cargo ships, and tankers have to undergo regular inspections at survey intervals. Up to now, this inspection is preformed by ship surveyors manually, using visual close-up surveys and thickness measurement [1]. The requirements for the vessel inspection are described in detail in [2]. Vessel inspection is performed usually every five years, depending on the requirements of the ship classification society. Ship surveyors have to be within arms reach of the inspection point in order to estimate structural damage, pitting, and corrosion. Based on the results of a first visual inspection, additional thickness measurements are performed. The inspec-tion data is recorded manually using cameras and clipboards during the inspection. Getting access to the inspection points is the most time consuming, and the most expensive part of the whole inspection process, because it takes several days or even weeks until the access can be provided using scaffolding or to provide access to the ship using "cherry-picking" methods. In the latter case, the surveyor is inside a basket, transported by a tower crane or a crane with a hydraulic arm. This method is potentially risky for the surveyor because the basket can collide with the structural parts of the ship, causing injuries to the surveyor. A single section of a cargo hold of an average vessel can easily reach 15 meters in height and more than 20 meters across. Figure 2 shows different cargo holds of a bulk carrier and a container ship. In order to reduce inspection time by removing the need for scaffolding and crane installation, a small magnetic climbing robot is able to provide visual data by climbing up bulkheads and recording images from the robot position. Localization of the robot inside the ship is mandatory in order to relocate and to classify damages of the ship. Inside a large cargo hold, as depicted in Figure 2, 


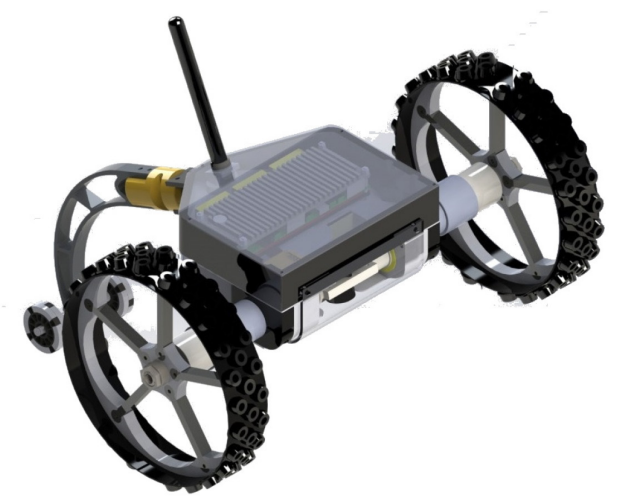

Fig. 1. CAD rendering of the Marine Inspection Robotic Assistant (MIRA) robot.

a flying inspection system is a suitable platform due to the large space within such a hold. UAVs which were recently developed for ship inspection purposes are described in [3] and [4]. Flying systems are suitable for a quick overview of the structures inside a hull or a ship superstructure. In some cases, the surveyor needs a visual close-up survey in order to classify a defect. Besides the visual data provided by an inspection service robot, thickness measurements are usually taken. For such an application, a flying system is not suitable because it cannot approach a bulkhead or structure because of possible collisions.

Within the INCASS project, a fleet of three inspection robots is proposed which are applied during three discrete stages of the inspection process. Based on the inspection requirements, the adequate locomotion principle for the in-spection robot is based on magnets. Some magnetic climbing systems for ship inspection exist in research and some are already commercially available. Two examples of a magnetic inspection robots are presented in [5] and [6]. Both robots use magnetic tracks which provide sufficient adhesion force on metal surfaces.

Inside ballast water tanks and inside narrow structures, a low-cost inspection system was introduced using a railway system [7]. Other inspection systems, especially for inspection inside tanks and ballast water tanks, were developed within the ROTIS II project [8]. Recently developed systems, such as

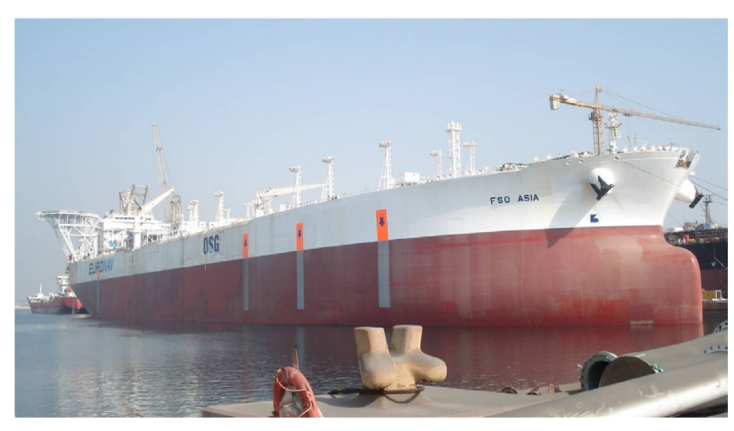

(a)

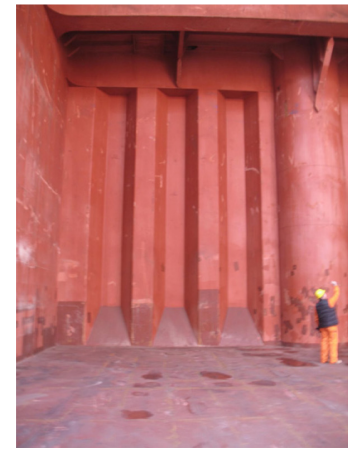

(b)

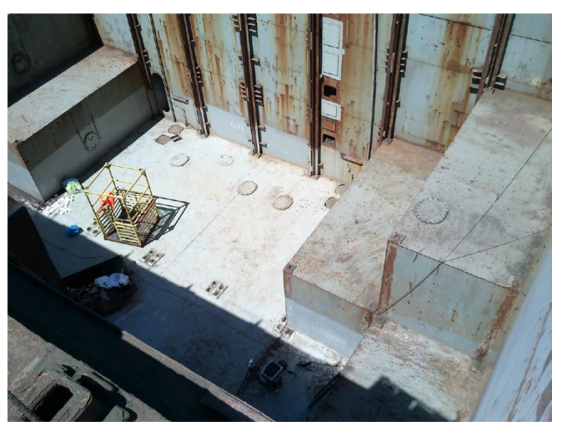

(c)

Fig. 2. (a) Marine vessels have to undergo regular inspections. (b) Cargo hold of a bulk carrier. (c) Cargo hold of a container vessel.

the AUV Dagon [9], are usable for the underwater inspection of ship hulls under water and for the propeller section.

For dry inspection of cargo holds, climbing robots are the most suitable systems, because they can transport sensors, such as cameras or sonar thickness measurements, close to the inspected area. The inspection area is usually several meters up on a bulkhead. Traditionally, the inspection is done by surveyors using staging and "cherry-picking" methods, which are costly and time consuming, and sometimes dangerous due to the low safety standards in some countries. Because marine 
vessels are made of steel, magnetic climbing loco-motion is a suitable way to move around the cargo hold of a vessel. Most climbing robots are heavy-weight systems and need an additional safety measure, such as a safety rope. In some scenarios, a safety rope is not usable due to the dangers of entanglement. In this paper we introduce a magnetic climbing robot MIRA which is a semi-autonomous and improved version of the lightweight crawler described in [10]. The former version was purely tele-operated and did not provide on-board control capabilities. The new robot has an internal embedded CPU which enables the system to execute basic inspection behaviors, such as weld seam following and collision avoidance based on the integrated camera. Figure 1 shows the 3D CAD design of the robot.

\section{HARDWARE AND SOFTWARE ARCHITECTURES}

The robot MIRA is the second generation and successor of the MINOAS crawler [10]. In this improved version, the remote-control transmission unit (RC) is replaced with a gumstix-robovero embedded system board which enables the crawler to be steered over WiFi or partly autonomous by the board itself.

Due to the removal of the RC the motors are now controlled with the Orion Robotics RoboClaw motor controller [11], which enables us to use the additional data of the motor encoders. The fixed camera of the first magnet crawler is upgraded to a tiltable camera unit which is directly connected to the embedded gumstix board. This gives the user a better possibility to look on one hand on its way and on the other hand directly under the crawler, to inspect the desired surface. Structure and materials of the front wheels and the back wheel had to be changed to improve its robustness. Due to these changes the weight increased from $964 \mathrm{~g}$ to about $1125 \mathrm{~g}$.

\section{A. Hardware}

MIRA is equipped with all necessary resources to behave as an autonomous vehicle. The system is supplied with an em-bedded computing system composed of the Robovero Gumstix expansion board [12] and the Gumstix embedded Computer- on-Module (COM) Overo AirSTORM COM [13] that initially used Ubuntu Linaro Linux [14]. MIRA has WiFi, Bluetooth as communication alternatives. Furthermore, it includes other sensors, such as an inertial measurement unit (IMU) and temperature sensors. Some of these sensors will be visualized through a graphical user interface (GUI) that is part of the remote station of the robot.

Robovero is an electronic board for robotic applications and the main board of the robot control unit. Some of its most important features are the inclusion of a microcontroller, a 9-DoF IMU (3DoF gyroscope, 3-DoF accelerometer, 3- DoF compass), power electronics to connect motors, and a USB HUB. Robovero has a firmware that permits the micro-controller to receive commands through a USB connection. The commands allow us to read and write I/O devices (such as I2C, UARTs, SPI, PWM, A/D, etc.). Therefore, Robovero itself is not an embedded computing system, but can be considered a peripheral board. However, microntroller commands are received through USB, either from an external computer or from the embedded one (Overo AirSTORM). Finally, the 


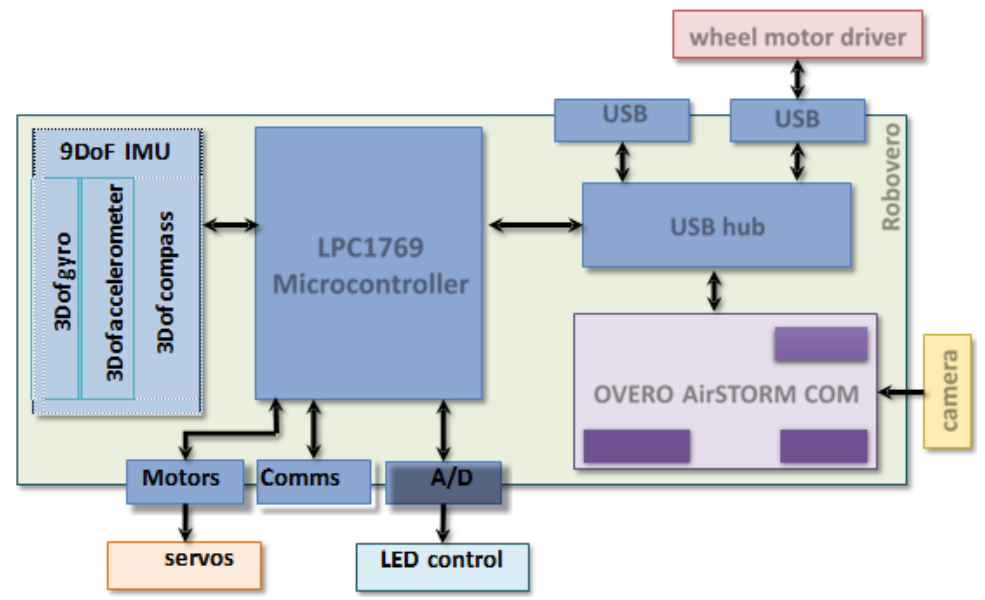

Fig. 3. The hardware architecture with the electronic boards connecting the main components of processors, sensors, and actuators.

whole system (Robovero and Overo) is an embedded com-puting system which, together with the actuators and sensors, complete the hardware architecture of the system (see Fig. 3).

\section{B. SOFTWARE}

The software system architecture can be divided into two main parts. A high-level computation layer must interpret primitive movement commands in teleoperation mode and generate the respective actuators commands or, alternatively, to navigate autonomously according to high-level orders and the information provided by the sensors. On the other hand, there is a lowlevel computation layer that is in charge of collecting (reading) information from sensors and to generate low-level control commands to the actuator driver hardware. Both high and low layers are directly related to hardware ar-chitecture, as the high-level corresponds to Overo AirSTORM programming, while the low-level corresponds to Robovero microcontroller (see Fig. 4).
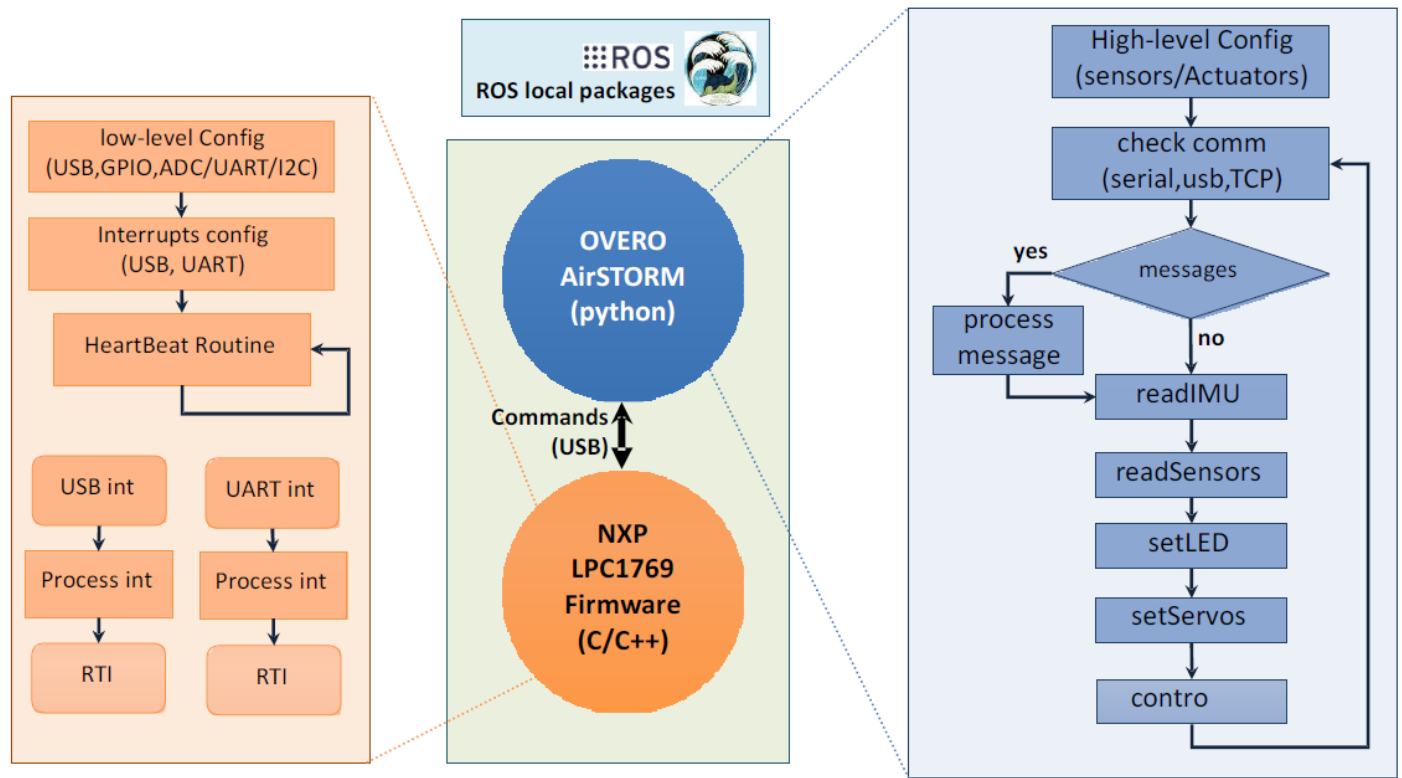

Fig. 4. The software architecture that presents the low-level and high-level computation layers

The high-level functionallity and control of the robot are implemented in ROS packages (see Fig. 4). The Robot Oper-ating System (ROS) is a standard and open-source operating system for 
robot control [15]. ROS is not an operating system in the traditional sense of process management and scheduling; rather, it provides a structured communication layer above the host operating systems of a heterogeneous compute cluster. Our software ROS packages interact with each of the sub-systems in $\mathrm{C}++$ and Python for direct system control. The system described uses a Linux based software framework as an operating system (OS) for providing the advantages of using an OS which supports developing additional modules that can be easily implemented and integrated. ROS provides operating system like service for the robot. It is a meta-operating system which loads on top of an operating system to provide a standardized set of software framework and APIs. These facilities cannot only help robots but also other embedded systems with a rich set of tools to successfully manage the complexity. With ROS handeling the basic communications and data exchange, the different packages implement the control and sensor reading as depicted in Fig. 6.

\section{MECHANICAL DESIGN}

Due to the new desired abilities of MIRA, major com-ponents of the first generation robot had to be changed and redesigned. Now the front wheel magnets are enclosed in a polyurethane structure except of the contact surface (see Figure 4). The wheel itself is changed from polyacetal to polyurethane, to make it more robust against shocks and prevent it from breaking. The polyurethane wheel is able to transform the impact energy into temporary deformation. The magnet arrangement of the wheels is also modified and the number of magnets are increased to handle the additional weight.

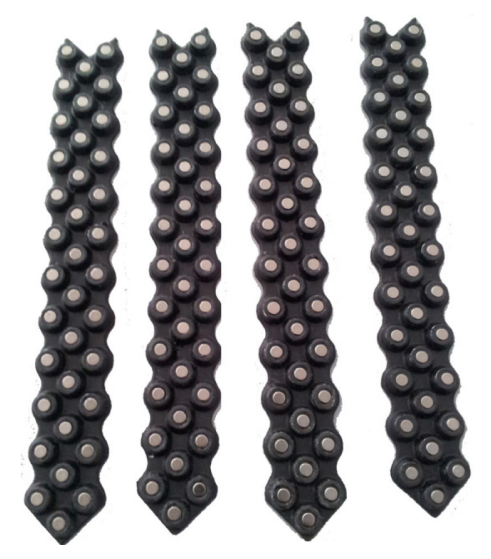

Fig. 5. New flexible concept for the magnet-wheel integration, based on polyurethane casting.

The rear wheel is changed from a single easy breaking ring magnet to a combination of rubber and segmented ring magnets. The rubber enables the construction to deform on impact and lowers the risk of breaking the magnet. If a magnet still breaks, not the whole ring has to be changed, only the damaged segment.

The rigid core structure of the crawler is designed out of $1 \mathrm{~mm}$ carbon-fiber plates (CFK) and connected by polyacetal elements (the motor and tail elastic joint). The plates arrange motor and tail position and also carry the sensitive parts like: embedded system, motor driver, LiPo-battery, LiPosaver and the tilt camera. The upper body casing and some fastening elements are printed in acrylonitrile butadiene styrene (ABS) synthetics. The 3D printing of ABS has two major advantages: ABS is a durable and impact resistant material and the printing method allows manual adjustments of density of chosen areas. This is a good feature for light weight construction. The main covering parts of the crawler are designed from $1 \mathrm{~mm}$ aluminum sheets (but can be changed to CFK if desired). The aluminum casing is chosen because in case of a crash it is more ductile than 
the CFK type but at the price of a slightly higher weight. Sensitive structures like the robovero and the motor driver boards are additionally encased with a modular glass fiber reinforced plastics (GFK) plates.

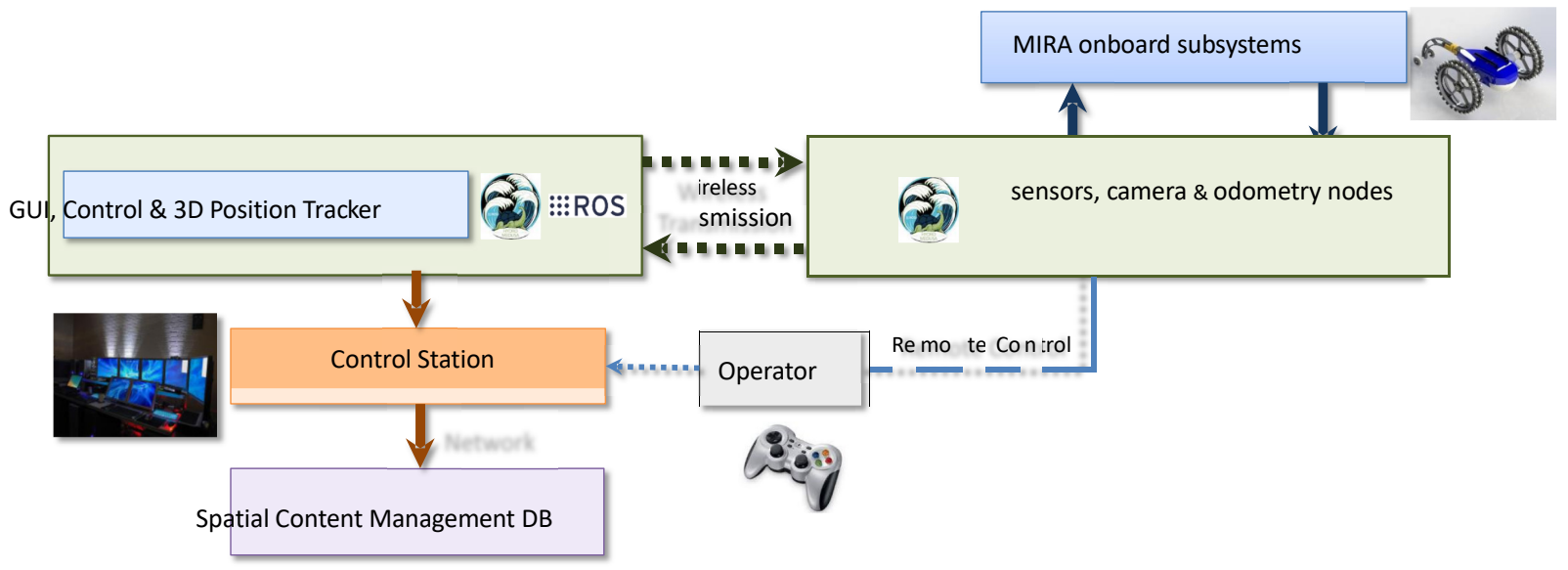

Fig. 6. Overall architecture of the MIRA lightweight inspection system. In addition to the operator as manin-the-loop, the system architecture is comprised of the mobile robot, the local control station, and the backend connection to the decisions support system database.

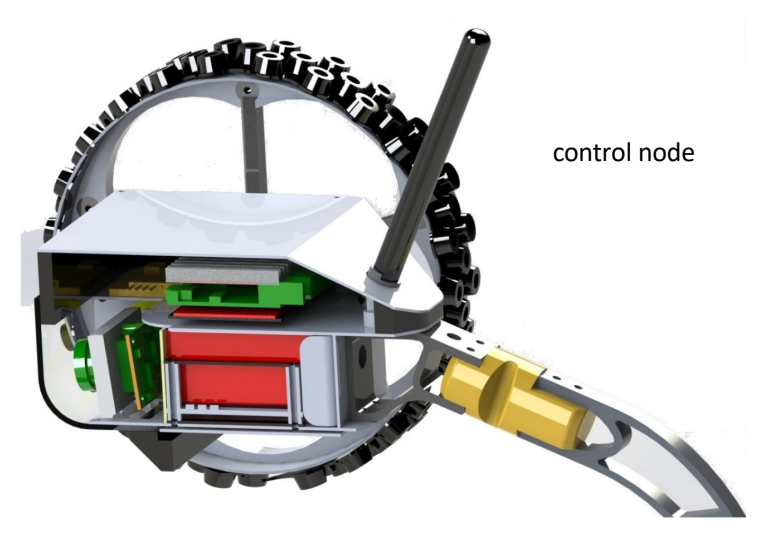

Fig. 7. Sectional view of the MIRA robot.

\section{CONCLUSION}

This paper presents a system description and the main aspects related to the design, construction, and implementation of MIRA, a lightweight magnetic climbing robot for ship inspection. The robot combines the reliability of the wheeled robot with the flexibility and versatility required to operate in different types of ship bulkheads. Due to this, it turns out to be an excellent candidate to be a part of a heterogeneous robotic team for ship inspection.

These features are mainly achieved due to its original movement based on magnetic traction forces instead of using friction-based movement. Additionally, its shape and weight prevent MIRA from damaging the environment or people, being able to continue with its moving and sensing capabilities after collisions or even small falls. However, more tests and experiments to improve and validate the design and sensor performance are to be carried out in order to optimize the system performance.

Finally, some issues have to be addressed in order to fulfill fully autonomous operation and integration into a heteroge-neous system. in order to make the integration of MIRA into the whole inspection system easier, an effort is being carried out so as to provide it with a standard 
connectivity by using the common robot operating system (ROS) framework. This task becomes easier since it natively works using Ubuntu as its operating system and encapsulate its functionality in ROS packages.

\section{ACKNOWLEDGMENT}

We thank all team members of the (INCASS) [16] who provided valuable comments, ideas, and assistance, which were essential to this study.

This work is developed for the project "Inspection Capa-bilities for Enhanced Ship Safety (INCASS)" and funded by the European Union - SEVENTH FRAMEWORK PROGRAMME (Activity code: SST.2013.4-2, Call: FP7-TRANSPORT-2013-MOVE-1, Grant agreement Nr.: 605200).

\section{References}

[1] K. Tanneberger and A. Grasso, "MINOAS Deliverable (D1): Definition of the Inspection Plan / Definition of Acceptance Criteria," http://minoasproject.eu/excibition/Publications/PDF/D1.pdf, 2011. [On-line]. Available: http://minoasproject.eu/excibition/Publications/PDF/ D1.pdf

[2] M. Eich, F. Bonnin-Pascual, E. Garcia-Fidalgo, A. Ortiz, G. Bruzzone, Y. Koveos, and F. Kirchner, "A Robot Application to Marine Vessel Inspection," Journal of Field Robotics, vol. 31, no. 2, pp. 319-341, 2014

[3] A. Ortiz, F. Bonnin-Pascual, and E. Garcia-Fidalgo, "On the Use of UAVs for Vessel Inspection Assistance," in Proceedings of the 1st Workshop on Research, Education and Development on Unmanned Aerial Systems, Seville (Spain), Nov 30th - Dec 1st, 2011, pp. 71-80.

[4] F. Ortiz Albertoand Bonnin-Pascual, E. Garcia-Fidalgo, and J. P. Bel-tran, "A Control Software Architecture for Autonomous Unmanned Vehicles Inspired in Generic Components (I)," in Proc. 19th Mediter-ranean Conf. Control and Automation, 2011.

[5] M. Bibuli, G. Bruzzone, G. Bruzzone, M. Caccia, M. Giacopelli, A. Petitti, and E. Spirandelli, "MARC: Magnetic Autonomous Robotic Crawler Development and Exploitation in the MINOAS Project," in Proc. 11th International Conference on Computer and IT Applications in the Maritime Industries, COMPIT 2012, 2012. [Online]. Available: http://www.ssi.tu-harburg.de/doc/webseiten dokumente/compit/dokumente/Proceeding Compit2012 Liege.pdf

[6] L. P. Kalra, J. Guf, and M. Meng, "A Wall Climbing Robot for Oil Tank Inspection,” in Proceedings of the International Conference on Robotics and Biomimetics (ROBIO), 2006, pp. $1523-1528$.

[7] L. Christensen, N. Fischer, S. Kroffke, J. Lemburg, and R. Ahlers, "Cost-Effective Autonomous Robots for Ballast Water Tank Inspection," Journal of Ship Production and Design, vol. 27, no. 3, pp. 127-136, 2011.

[8] ROTIS2, "ROTISII: Publishable Final Activity Report," http:/cordis.europa.eu/documents/ documentlibrary/124772341EN6.pdf, [Date of access: 10/12/2012]. [Online]. Available: http://cordis.europa.eu/documents/documentlibrary/124772341EN6.pdf 
[9] M. Hildebrandt, C. Gaudig, L. Christensen, S. Natarajan, P. M. Paran-hos, and J. Albiez, "Two years of Experiments with the AUV Dagon -a Versatile Vehicle for High Precision Visual Mapping and Algorithm Evaluation," in Proceedings of IEEE/OES Autonomous Underwater Vehicles. IEEE/OES Autonomous Underwater Vehicles (AUV-2012), September 24-27, Southampton, United Kingdom. o.A., 2012.

[10] M. Eich and T. Vogele, "Design and Control of a Lightweight Magnetic Climbing Robot for Vessel Inspection," in Proc. 19th Mediterranean Conf. Control and Automation, 2011.

[11] Roboclaw 2x5A Motor Controller, "http://www.orionrobotics.com/ Roboclaw-2x5A-MotorController p 270.html."

[12] gumstix Robotics Development Kit (RoboVero), "https://store.gumstix. com/index.php/products/504/."

[13] gumstix Overo AirSTORM COM, “https://store.gumstix.com/index. php/products/266.”

[14] Linaro Ubuntu Linux kernel and build for embedded ARM, "http:// www.linaro.org."

[15] S. Cousins, "Exponential growth of ros [ros topics]," Robotics Automa-tion Magazine, IEEE, vol. 18, no. 1, pp. 19-20, March 2011.

[16] Inspection Capabilities for Enhanced Ship Safety (INCASS) project, "http://www.incass.eu.” 